\title{
A New Hybrid Implicit-Explicit FDTD Method for Local Subgridding in Multiscale 2D-TE Scattering Problems
}

\author{
A. Van Londersele, D. De Zutter, Fellow, IEEE, D. Vande Ginste, Senior Member, IEEE
}

\begin{abstract}
The conventional Finite-Difference Time-Domain (FDTD) method with staggered Yee scheme does not easily allow including thin material layers, especially so if these layers are highly conductive. This paper proposes a novel subgridding technique for 2D problems, based on a Hybrid Implicit-Explicit (HIE) scheme, that efficiently copes with this problem. In the subgrid, the new method collocates field components such that the thin layer boundaries are defined unambiguously. Moreover, aspect ratios of more than a million do not impair the stability of the method and allow for very accurate predictions of the skin effect. The new method retains the Courant limit of the coarse Yee grid and is easily incorporated into existing FDTD codes. A number of illustrative examples, including scattering by a metal grating, demonstrate the accuracy and stability of the new method.
\end{abstract}

Index Terms-Finite-Difference Time-Domain (FDTD), Hybrid Implicit-Explicit (HIE), multiscale, scattering, subgridding

\section{INTRODUCTION}

Due to the increasing impact of parallel processing, the Finite-Difference Time-Domain (FDTD) method remains one of the most popular computational methods for solving Maxwell's equations. Besides its simplicity associated with its regular grid, it also has some clear advantages such as its broadband nature and its ability to treat nonlinearities.

FDTD suffers, however, from the Courant-Friedrichs-Lewy (CFL) stability criterion which, together with the numerical dispersion error, causes the CPU-time to become exceedingly large in cases where geometrical features are involved that are orders of magnitude smaller than the working wavelength. This well-known problem has been the subject in a lot of recent research. A possible solution involves the application of subgridding techniques with a local time step, bounded by a local stability criterion. Despite some important work in this domain [1]-[5], an accurate, stable and completely explicit version of such a refinement scheme has, to our knowledge, still not been found.

Another way is to relax the CFL limit by systematically filtering out the high wave numbers at every iteration [6]. This technique is easily incorporated into existing FDTD codes, but the time won by relaxing the CFL limit should not be wasted by extra computations. In fact, applying a filter

The authors are with the Electromagnetics Group, Department of Information Technology, Ghent University, 9000 B-Gent, Belgium (e-mail: arne.vanlondersele@ugent.be). implies the calculation of time-consuming Fourier and inverseFourier transforms. Spatial filtering is also used to stabilize subgridding schemes [7]. At least one of the fields in the dense grid is spatially filtered in order to equalize the time step of the dense and coarse grid. This results in one global time step determined by the cell size of the coarse grid. However, reasonably large aspect ratios, for instance 1:1000 in one dimension, seem to be unreachable for the spatially-filtered subgridding scheme as it requires discarding too many wave numbers.

Yet an alternative approach to tackle the multiscale problem has recently been proposed in [8]. This method does not modify the field solutions, but searches the space of stable eigenmodes of the discrete curl-curl operator. Then, at any time, the field solution $E(t)$ can be calculated as a superposition of these stable eigenmodes, i.e. $E(t)=Q_{s t} y(t)$ with $Q_{s t}$ the matrix composed of all stable eigenmodes. The unknown coefficient vector $y(t)$ is discretized in time resulting in explicit update equations which are unconditionally stable. The time step is chosen based on accuracy. The method is however irreconcilable with conventional FDTD.

Other procedures circumvent the CFL limit by using implicit time integration instead of the classical leapfrog scheme, which leads to unconditionally stable methods that require the inversion of sparse matrices. Examples of these implicit methods are Crank-Nicolson (CN) FDTD [9], alternating direction implicit (ADI) FDTD [10], locally one-dimensional (LOD) FDTD [11,12] and Laguerre-FDTD [13,14]. A lot of comparisons concerning the CPU-time and accuracy of these implicit schemes have been reported [15]-[17]. Compared to explicit methods, they all reduce the number of time steps per simulation at the expense of a higher computational effort per time step. For multiscale configurations, optimal performance is obtained by applying the fast (explicit and parallel) FDTD method in the bulk part of the simulation domain together with an implicit method to resolve the fine geometries. Since the time step of the implicit method can be chosen arbitrarily, it is set equal to the FDTD time step of the coarse grid. In order to match both grids, one can use simple spatial interpolations $[18,19]$ or, alternatively, one can transfer the information between both grids through equivalent Huygens' surface currents [20].

Besides these purely implicit methods, whether or not combined with conventional FDTD in a multigrid configuration, another kind of (partially) implicit schemes exist: the so-called Hybrid Implicit-Explicit (HIE) FDTD methods [21,22]. They 
are used to analyse structures that are fine in at least one but not all directions. Therefore, the update equations consist of a mixture of implicit and explicit equations. As a consequence, they obey a reduced stability constraint.

The method advocated in this paper is a new twodimensional (2D) HIE-FDTD method for transverse electric (TE) waves dealing with geometrical structures that are fine in one dimension. The method is used for subgridding purposes and its grid, which uses a discretization that differs from the staggered Yee cell, is matched to the conventional FDTD grid with negligible spurious reflection. The proposed HIEsubgridding method allows extremely high aspect ratios, say more than $10^{6}$, and it is easily incorporated in conventional FDTD. The HIE scheme leverages some ideas reported in [23]-[25], where a fully collocated and unconditionally stable implicit method to simulate electromagnetic waves in fusion plasmas was presented. In [24], this fully collocated implicit method is combined with classical FDTD to obtain a 1D local refinement scheme. The HIE-FDTD method described here is the extension of this preliminary work to two dimensions.

This paper is organized as follows. Section II details the new HIE-FDTD method's formalism. Section III investigates the numerical dispersion and stability of the technique. In Section IV, several numerical scattering examples are listed to demonstrate the accuracy of the new subgridding method, including scattering by a metal grating. Finally, this paper ends with a concise discussion of the main findings in Section V.

\section{Description OF THE NEW HIE METHOD}

We restrict the discussion to the 2D-TE case. Extension to the 2D-TM case runs along the same lines, but discussing both simultaneously would impair the clarity of the explanation. Extension to 3D remains the topic for further research.

In a general sourceless, piecewise homogeneous medium, the TE-problem is described by

$$
\begin{aligned}
\frac{\partial E_{x}}{\partial y}-\frac{\partial E_{y}}{\partial x} & =\frac{\partial B_{z}}{\partial t} \\
\mu^{-1} \frac{\partial B_{z}}{\partial y} & =\varepsilon \frac{\partial E_{x}}{\partial t}+\sigma E_{x} \\
-\mu^{-1} \frac{\partial B_{z}}{\partial x} & =\varepsilon \frac{\partial E_{y}}{\partial t}+\sigma E_{y},
\end{aligned}
$$

where all fields lie in the $x y$-plane and are invariant in the $z$-direction. The typical configuration we want to tackle is depicted in Fig. 1. A thin, e.g. highly conductive, slab is embedded in a classical FDTD grid. This slab can be substantially smaller than the Yee cell of the global FDTD grid in one dimension, here the $x$-dimension, i.e. $d \ll x_{2}-x_{1}=\Delta x$, although, it comprises several Yee cells in the other dimension. In what follows, the new HIE-subgridding method is presented as the result of a Petrov-Galerkin method. To this end, suitable basis and testing functions are constructed from onedimensional pulse and triangle functions. First, the standard FDTD method in the FDTD grid (i.e. the area outside PQRS in Fig. 1) is treated in a similar fashion as [3], starting from the well-known Whitney forms. Then, the HIE update equations inside PQRS are derived based on the insights from [23] and, finally, the interface condition between both grids

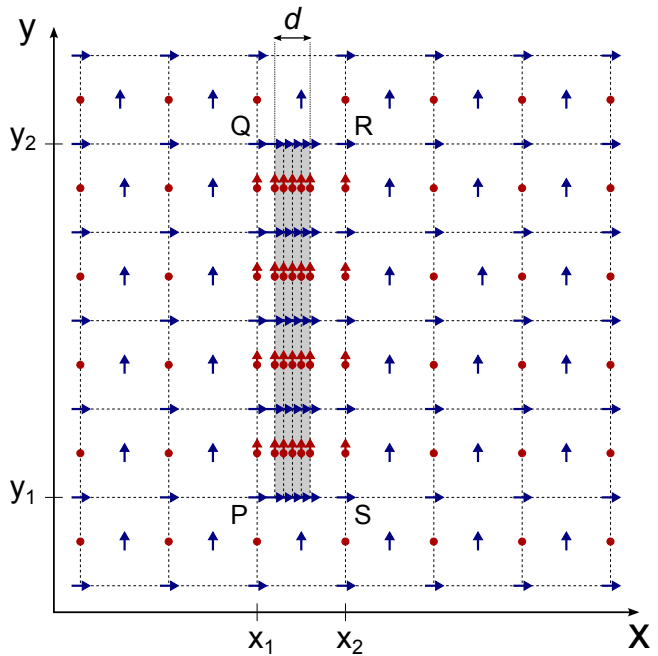

Fig. 1: The HIE-subgridding scheme for a vertically oriented slab. The rectangle PQRS encloses the HIE region which consists of a thin conductive slab of width $d$ and a padding area filled with the same medium as the surrounding FDTD grid. The arrows indicate the discretization points of the electric field components, the dots those of the magnetic field. The blue markers are discretized at integer multiples of $\Delta t$, whereas the red markers are discretized at halfinteger multiples of $\Delta t$.

is established. It is important to already draw attention to the fact that the HIE grid is partially collocated. Compared to the conventional FDTD discretization scheme, the electric field's $y$-component, tangential to the thin slab, is shifted to the spatial and temporal position of $B_{z}$. This gives rise to a discretization scheme where all field components are ordered in layers parallel to the slab surface. The major advantage of this approach is that no uncertainty as to the thickness of the thin slab is introduced, as would be the case with a staggered approach.

\section{A. Classical leapfrog 2D-TE equations}

We use the 2D-TE Yee cell, with dimensions $\Delta x \times \Delta y$, as shown in Fig. 2 (left). The $E_{x}, E_{y}$ and $B_{z}$ field components are expanded as

$$
\begin{aligned}
& E_{x}(x, y, t)=\sum_{i, j, n} E_{x, i+\frac{1}{2}, j}^{n+\frac{1}{2}} \Pi_{i+\frac{1}{2}}(x) \Lambda_{j}(y) \Pi_{n+\frac{1}{2}}(t) \\
& E_{y}(x, y, t)=\sum_{i, j, n} E_{y, i, j+\frac{1}{2}}^{n+\frac{1}{2}} \Lambda_{i}(x) \Pi_{j+\frac{1}{2}}(y) \Pi_{n+\frac{1}{2}}(t) \\
& B_{z}(x, y, t)=\sum_{i, j, n} B_{z, i+\frac{1}{2}, j+\frac{1}{2}}^{n} \Pi_{i+\frac{1}{2}}(x) \Pi_{j+\frac{1}{2}}(y) \Lambda_{n}(t) .
\end{aligned}
$$

For the $x$-dependence, the pulse and triangle basis functions in (4)-(6) are defined as

$$
\begin{aligned}
\Pi_{i+\frac{1}{2}}(x) & = \begin{cases}1 & i \Delta x<x<(i+1) \Delta x \\
0 & \text { elsewhere }\end{cases} \\
\Lambda_{i}(x) & = \begin{cases}1-i+\frac{x}{\Delta x} & (i-1) \Delta x<x \leq i \Delta x \\
1+i-\frac{x}{\Delta x} & i \Delta x<x<(i+1) \Delta x \\
0 & \text { elsewhere }\end{cases}
\end{aligned}
$$


and similarly for the $y$ - and $t$-dependence. The weights in the summations (4)-(6) are the actual field components as found in the classical FDTD derivation, i.e.

$$
\begin{aligned}
E_{x, i+\frac{1}{2}, j}^{n+\frac{1}{2}} & =E_{x}((i+1 / 2) \Delta x, j \Delta y,(n+1 / 2) \Delta t) \\
E_{y, i, j+\frac{1}{2}}^{n+\frac{1}{2}} & =E_{y}(i \Delta x,(j+1 / 2) \Delta y,(n+1 / 2) \Delta t) \\
B_{z, i+\frac{1}{2}, j+\frac{1}{2}}^{n} & =B_{z}((i+1 / 2) \Delta x,(j+1 / 2) \Delta y, n \Delta t),
\end{aligned}
$$

with $\Delta x, \Delta y$ and $\Delta t$ the space and time increments. The expansions (4)-(6) are the so-called Whitney forms. They possess the curl inclusion property. The curl operator projects the space of one-forms such as $E_{x}$ and $E_{y}$ exactly on the space of 2-forms such as $B_{z}$. Assuming weak differentiability, this can be seen from the fact that

$$
\frac{\mathrm{d}}{\mathrm{d} x} \Lambda_{i}=\frac{1}{\Delta x}\left(\Pi_{i-\frac{1}{2}}-\Pi_{i+\frac{1}{2}}\right) \text {. }
$$

Substituting the expansions (4)-(6) into Faraday's law (1), using the property (12), all terms can be expressed using solely pulse basis functions. Hence, Faraday's law is readily satisfied if terms in the same pulse $\times$ pulse $\times$ pulse expansion are identified on both sides of the equation. For the terms containing

$$
\Pi_{k+\frac{1}{2}}(x) \Pi_{l+\frac{1}{2}}(y) \Pi_{m+\frac{1}{2}}(t),
$$

this results in the classical FDTD relationship

$$
\begin{aligned}
& \frac{1}{\Delta t}\left(B_{z, k+\frac{1}{2}, l+\frac{1}{2}}^{m+1}-B_{z, k+\frac{1}{2}, l+\frac{1}{2}}^{m}\right)= \\
& \frac{1}{\Delta y}\left(E_{x, k+\frac{1}{2}, l+1}^{m+\frac{1}{2}}-E_{x, k+\frac{1}{2}, l}^{m+\frac{1}{2}}\right)-\frac{1}{\Delta x}\left(E_{y, k+1, l+\frac{1}{2}}^{m+\frac{1}{2}}-E_{y, k, l+\frac{1}{2}}^{m+\frac{1}{2}}\right) .
\end{aligned}
$$

Since the pulse functions in (4)-(6) are not differentiable, one cannot enforce Ampère's law in the same way as Faraday's law. We now substitute (4) and (6) into (2) and evaluate this equation in the weak sense by multiplying left- and right-hand side with the testing function

$$
T_{k+\frac{1}{2}, l}^{m}(x, y, t)=\Pi_{k+\frac{1}{2}}(x) \Lambda_{l}(y) \Lambda_{m}(t),
$$

followed by integration over the simulation domain. The derivatives of the pulse functions are transferred to the testing functions via integration by parts. The remaining integrals are evaluated using a trapezoidal integration rule, a process referred to as mass-lumping [3]. The final result is again the well-known classical FDTD relation, namely

$$
\begin{aligned}
& \frac{\mu^{-1}}{\Delta y}\left(B_{z, k+\frac{1}{2}, l+\frac{1}{2}}^{m}-B_{z, k+\frac{1}{2}, l-\frac{1}{2}}^{m}\right)= \\
& \quad \frac{\varepsilon}{\Delta t}\left(E_{x, k+\frac{1}{2}, l}^{m+\frac{1}{2}}-E_{x, k+\frac{1}{2}, l}^{m-\frac{1}{2}}\right)+\frac{\sigma}{2}\left(E_{x, k+\frac{1}{2}, l}^{m+\frac{1}{2}}+E_{x, k+\frac{1}{2}, l}^{m-\frac{1}{2}}\right) .
\end{aligned}
$$

A similar reasoning applied to (3) gives

$$
\begin{aligned}
& -\frac{\mu^{-1}}{\Delta x}\left(B_{z, k+\frac{1}{2}, l+\frac{1}{2}}^{m}-B_{z, k-\frac{1}{2}, l+\frac{1}{2}}^{m}\right)= \\
& \quad \frac{\varepsilon}{\Delta t}\left(E_{y, k, l+\frac{1}{2}}^{m+\frac{1}{2}}-E_{y, k, l+\frac{1}{2}}^{m-\frac{1}{2}}\right)+\frac{\sigma}{2}\left(E_{y, k, l+\frac{1}{2}}^{m+\frac{1}{2}}+E_{y, k, l+\frac{1}{2}}^{m-\frac{1}{2}}\right) .
\end{aligned}
$$

In the finite-element community, it is well understood that FDTD can be seen as a special case of the Finite-Element Time-Domain (FETD) method with a structured grid, Whitney forms and mass-lumping [3].

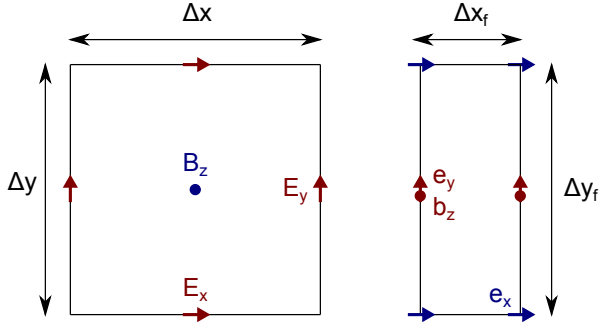

Fig. 2: Classical 2D-TE Yee cell (left) and new HIE cell for a thin slab extending in the $y$-dimension (right). The blue markers indicate field components discretized at integer multiples of $\Delta t$, the red markers at half-integer multiples.

\section{B. HIE 2D-TE equations for a thin conductive slab}

The right part of Fig. 2 shows the arrangement of the fields in a HIE cell for a layer which is thin in the $x$-direction. The $E_{x}, E_{y}$ and $B_{z}$ fields are now deliberately discretized at exactly the same $x$-coordinates as to avoid any ambiguity with respect to the thickness of the thin layer when aggregating cells in the $x$-direction. In the $y$-direction, staggering is retained. Moreover, the fine cell dimensions $\Delta x_{f} \times \Delta y_{f}$ are chosen such that $\Delta y_{f}=\Delta y$ (i.e. identical to the overall FDTD grid), with $\Delta x_{f}$ a fraction of the total thickness $d$ of the thin layer and with $\Delta x_{f} \ll \Delta x$. The time increment $\Delta t$ also remains identical to the time step in the overall FDTD grid. The expressions (4)-(6) are now replaced by

$$
\begin{aligned}
& E_{x}(x, y, t)=\sum_{i, j, n} e_{x, i, j}^{n} \Lambda_{i}(x) \Lambda_{j}(y) \Lambda_{n}(t) \\
& E_{y}(x, y, t)=\sum_{i, j, n} e_{y, i, j+\frac{1}{2}}^{n+\frac{1}{2}} \Lambda_{i}(x) \Pi_{j+\frac{1}{2}}(y) \Pi_{n+\frac{1}{2}}(t) \\
& B_{z}(x, y, t)=\sum_{i, j, n} b_{z, i, j+\frac{1}{2}}^{n+\frac{1}{2}} \Lambda_{i}(x) \Pi_{j+\frac{1}{2}}(y) \Pi_{n+\frac{1}{2}}(t) .
\end{aligned}
$$

A lowercase notation is adopted for the HIE expansion coefficients as this will be helpful to distinguish between the variables in the main grid and the subgrid later on. Both Faraday's and Ampère's law now only allow weak solutions. A similar analysis with mass-lumping readily shows that substitution of (18)-(20) into (2) and testing with

$$
T_{k, l}^{m+\frac{1}{2}}(x, y, t)=\Lambda_{k}(x) \Lambda_{l}(y) \Pi_{m+\frac{1}{2}}(t)
$$

leads to the explicit update equation

$$
e_{x, k, l}^{m+1}=\frac{\frac{\varepsilon}{\Delta t}-\frac{\sigma}{2}}{\frac{\varepsilon}{\Delta t}+\frac{\sigma}{2}} e_{x, k, l}^{m}+\frac{1}{\frac{\varepsilon}{\Delta t}+\frac{\sigma}{2}} \frac{\mu^{-1}}{\Delta y}\left(b_{z, k, l+\frac{1}{2}}^{m+\frac{1}{2}}-b_{z, k, l-\frac{1}{2}}^{m+\frac{1}{2}}\right) \text {. }
$$

Testing (1) and (3) with

$$
T_{k+\frac{1}{2}, l+\frac{1}{2}}^{m}(x, y, t)=\Pi_{k+\frac{1}{2}}(x) \Pi_{l+\frac{1}{2}}(y) \Lambda_{m}(t) .
$$

results in the implicit update equations

$$
\begin{aligned}
& \frac{1}{\Delta x_{f}}\left[\begin{array}{ll}
-1 & 1
\end{array}\right]\left[\begin{array}{c}
e_{y, k} \\
e_{y, k+1}
\end{array}\right]_{l+\frac{1}{2}}^{m+\frac{1}{2}}+\frac{1}{\Delta t}\left[\begin{array}{ll}
1 & 1
\end{array}\right]\left[\begin{array}{c}
b_{z, k} \\
b_{z, k+1}
\end{array}\right]_{l+\frac{1}{2}}^{m+\frac{1}{2}} \\
& =-\frac{1}{\Delta x_{f}}\left[\begin{array}{ll}
-1 & 1
\end{array}\right]\left[\begin{array}{c}
e_{y, k} \\
e_{y, k+1}
\end{array}\right]_{l+\frac{1}{2}}^{m-\frac{1}{2}}+\frac{1}{\Delta t}\left[\begin{array}{ll}
1 & 1
\end{array}\right]\left[\begin{array}{c}
b_{z, k} \\
b_{z, k+1}
\end{array}\right]_{l+\frac{1}{2}}^{m-\frac{1}{2}} \\
& \quad+\frac{1}{\Delta y}\left(e_{x, k, l+1}^{m}+e_{x, k+1, l+1}^{m}-e_{x, k, l}^{m}-e_{x, k+1, l}^{m}\right)
\end{aligned}
$$


and

$\left(\frac{\varepsilon}{\Delta t}+\frac{\sigma}{2}\right)\left[\begin{array}{ll}1 & 1\end{array}\right]\left[\begin{array}{c}e_{y, k} \\ e_{y, k+1}\end{array}\right]_{l+\frac{1}{2}}^{m+\frac{1}{2}}+\frac{\mu^{-1}}{\Delta x_{f}}\left[\begin{array}{ll}-1 & 1\end{array}\right]\left[\begin{array}{c}b_{z, k} \\ b_{z, k+1}\end{array}\right]_{l+\frac{1}{2}}^{m+\frac{1}{2}}$
$=\left(\frac{\varepsilon}{\Delta t}-\frac{\sigma}{2}\right)\left[\begin{array}{ll}1 & 1\end{array}\right]\left[\begin{array}{c}e_{y, k} \\ e_{y, k+1}\end{array}\right]_{l+\frac{1}{2}}^{m-\frac{1}{2}}-\frac{\mu^{-1}}{\Delta x_{f}}\left[\begin{array}{ll}-1 & 1\end{array}\right]\left[\begin{array}{c}b_{z, k} \\ b_{z, k+1}\end{array}\right]_{l+\frac{1}{2}}^{m-\frac{1}{2}}$.

By way of example, a more detailed derivation of (25) is given in Appendix A. Combining the two implicit equations (24) and (25) at all spatial positions, gives one big matrix equation

$$
\begin{aligned}
& {\left[\begin{array}{cc}
\frac{1}{\Delta x_{f}} A_{D} & \frac{1}{\Delta t} A_{I} \\
\left(\frac{\varepsilon}{\Delta t}+\frac{\sigma}{2}\right) A_{I} & \frac{\mu^{-1}}{\Delta x_{f}} A_{D}
\end{array}\right] X^{m+\frac{1}{2}}} \\
& =\left[\begin{array}{cc}
-\frac{1}{\Delta x_{f}} A_{D} & \frac{1}{\Delta t} A_{I} \\
\left(\frac{\varepsilon}{\Delta t}-\frac{\sigma}{2}\right) A_{I} & -\frac{\mu^{-1}}{\Delta x_{f}} A_{D}
\end{array}\right] X^{m-\frac{1}{2}}+\frac{1}{\Delta y}\left[\begin{array}{c}
Y^{m} \\
0_{N_{x} \times N_{y}}
\end{array}\right]
\end{aligned}
$$

Here, $X^{m+\frac{1}{2}}$ is a $\left[\left(2 N_{x}+2\right) \times N_{y}\right]$-dimensional matrix containing all unknown discrete field quantities for $E_{y}$ and $B_{z}$ inside the HIE grid, namely

$$
X^{m+\frac{1}{2}}=\left[\begin{array}{ccc}
e_{y, 0, \frac{1}{2}}^{m+\frac{1}{2}} & \cdots & e_{y, 0, N_{y}-\frac{1}{2}}^{m+\frac{1}{2}} \\
\vdots & \ddots & \vdots \\
e_{y, N_{x}, \frac{1}{2}}^{m+\frac{1}{2}} & \cdots & e_{y, N_{x}, N_{y}-\frac{1}{2}}^{m+\frac{1}{2}} \\
b_{z, 0, \frac{1}{2}}^{m+\frac{1}{2}} & \cdots & b_{z, 0, N_{y}-\frac{1}{2}}^{m+\frac{1}{2}} \\
\vdots & \ddots & \vdots \\
b_{z, N_{x}, \frac{1}{2}}^{m+\frac{1}{2}} & \cdots & b_{z, N_{x}, N_{y}-\frac{1}{2}}^{m+\frac{1}{2}}
\end{array}\right],
$$

where $N_{x}$ and $N_{y}$ denote the number of cells in the HIE region in the $x$ - and the $y$-dimension respectively. The $N_{x} \times N_{y}$ dimensional matrix $Y^{m}$ takes the contribution of $\frac{\partial E_{x}}{\partial y}$ into account, originating from Faraday's law in discrete form (24), i.e.

$$
\begin{gathered}
Y^{m}=\left[\begin{array}{ccc}
e_{x, 0,1}^{m} & \cdots & e_{x, 0, N_{y}}^{m} \\
\vdots & \ddots & \vdots \\
e_{x, N_{x}-1,1}^{m} & \cdots & e_{x, N_{x}-1, N_{y}}^{m}
\end{array}\right]+\left[\begin{array}{ccc}
e_{x, 1,1}^{m} & \cdots & e_{x, 1, N_{y}}^{m} \\
\vdots & \ddots & \vdots \\
e_{x, N_{x}, 1}^{m} & \cdots & e_{x, N_{x}, N_{y}}^{m}
\end{array}\right] \\
-\left[\begin{array}{ccc}
e_{x, 0,0}^{m} & \cdots & e_{x, 0, N_{y}-1}^{m} \\
\vdots & \ddots & \vdots \\
e_{x, N_{x}-1,0}^{m} & \cdots & e_{x, N_{x}-1, N_{y}-1}^{m}
\end{array}\right]-\left[\begin{array}{ccc}
e_{x, 1,0}^{m} & \cdots & e_{x, 1, N_{y}-1}^{m} \\
\vdots & \ddots & \vdots \\
e_{x, N_{x}, 0}^{m} & \cdots & e_{x, N_{x}, N_{y}-1}^{m}
\end{array}\right]
\end{gathered}
$$

$A_{D}$ and $A_{I}$ denote the differentiator and interpolator matrix respectively, both with dimension $N_{x} \times\left(N_{x}+1\right)$.

$$
A_{D}=\left[\begin{array}{ccccc}
-1 & 1 & 0 & 0 & \cdots \\
0 & -1 & 1 & 0 & \cdots \\
0 & 0 & -1 & 1 & \cdots \\
\vdots & \vdots & \vdots & \vdots & \ddots
\end{array}\right] \quad A_{I}=\left[\begin{array}{ccccc}
1 & 1 & 0 & 0 & \cdots \\
0 & 1 & 1 & 0 & \cdots \\
0 & 0 & 1 & 1 & \cdots \\
\vdots & \vdots & \vdots & \vdots & \ddots
\end{array}\right]
$$

The matrix equation (26) is still incomplete. It forms an underdetermined system since for each value of the $y$-index $l$, there are $2 N_{x}+2$ unknowns but only $N_{x}$ linearly independent equations. The remaining two rows, which have to be added to (26), will be found below by deriving the correct interface condition between the HIE grid and the surrounding FDTD grid (Section II-C).

We draw the reader's attention to the elegance of (26). It solely requires the inversion of one matrix which scales independently of $N_{y}$. The explicit calculation of this matrix inverse must however be avoided by applying an LU decomposition. This computation step can be optimized by first making the matrix banded, which is done by constructing a permutation matrix that rearranges $e_{y}$ and $b_{z}$ in $X^{m \pm \frac{1}{2}}$ according to their (increasing) $x$-coordinate. Every iteration step now only requires the solution of an upper- and lower triangular linear system, which can be performed very efficiently.

Looking back at Fig. 1, we note that the discretization in the $x$-direction inside PQRS is nonuniform as the increment along $x$ inside the conductive slab will typically be chosen much smaller than for the remaining padding space. This poses no problem to the implicit technique.

\section{Interface condition}

To derive the interface condition between the HIE and FDTD grid, the two grids first need to be aligned to each other. Having a closer look at Fig. 2, we see that by shifting the Yee cell a distance $\frac{\Delta x}{2}$ in the $x$-direction and a distance $\frac{\Delta t}{2}$ in the $t$-direction, the $E_{x}$ and $B_{z}$ discretization points of both schemes coincide on the interface. Thus, instead of using the standard Whitney form expansion (4)-(6), the shifted expansions

$$
\begin{aligned}
& E_{x}(x, y, t)=\sum_{i, j, n} E_{x, i, j}^{n} \Pi_{i}(x) \Lambda_{j}(y) \Pi_{n}(t) \\
& E_{y}(x, y, t)=\sum_{i, j, n} E_{y, i+\frac{1}{2}, j+\frac{1}{2}}^{n} \Lambda_{i+\frac{1}{2}}(x) \Pi_{j+\frac{1}{2}}(y) \Pi_{n}(t) \\
& B_{z}(x, y, t)=\sum_{i, j, n} B_{z, i, j+\frac{1}{2}}^{n+\frac{1}{2}} \Pi_{i}(x) \Pi_{j+\frac{1}{2}}(y) \Lambda_{n+\frac{1}{2}}(t)
\end{aligned}
$$

are adopted to integrate the HIE grid into the classical FDTD grid. Hence, the finite-difference relations (14), (16) and (17) remain valid apart from a small change of the indices $k$ and $m$. Two sets of interfaces remain:

- the left and right interfaces, which are affected by the implicit update of $e_{y}$ and $b_{z}$;

- the upper and lower interfaces, which are only affected by the explicit update of $e_{x}$.

The first set provides the extra information needed to add to (26) in order to obtain a unique solution for $e_{y}$ and $b_{z}$ at every update. The necessary interface conditions are obtained by testing Faraday's law (1) with the HIE testing function (23). At the right side ( $x=x_{2}$, see Fig. 1), this yields (30) as given below. Time $t_{0}=n \Delta t$ here stands for an arbitrary update moment in the overall FDTD grid, whereas $y_{0}=j \Delta y$ stands for the $y$-coordinate of grid points in the FDTD and HIE grids ranging between $y_{1}$ and $y_{2}-\Delta y$ (see Fig. 1). To further elucidate (30), consider Fig. 3 and Table I.

Table I summarizes the spatial and temporal position on which the three field components are discretized in both grids. $B_{z}$ and $E_{x}$ are treated in the same way in these grids, but this is not the case for $E_{y}$. Further note that all field values on the 


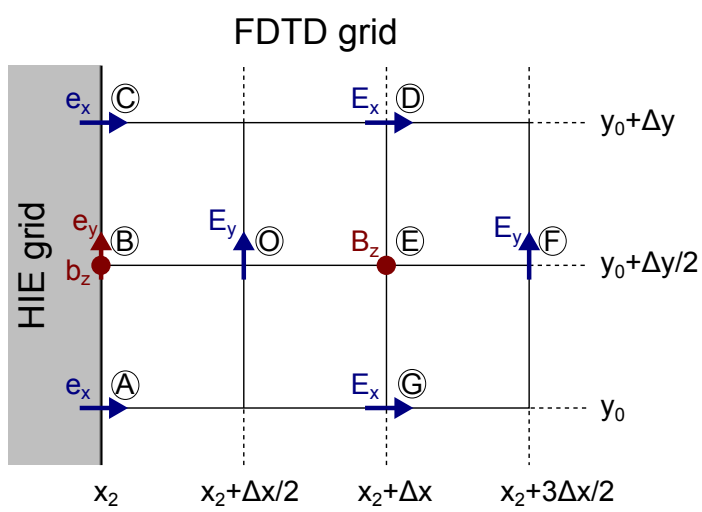

Fig. 3: Scheme relevant to the right interface condition (30) at $x=x_{2}$ in Fig. 1.

boundary of the subgrid are discretized according to the rules of the HIE grid. Fig. 3 zooms in on one particular $y_{0}$-value and all field values that play a role in (30). The first term in the 1.h.s. of (30) is the contribution of $\frac{\partial E_{x}}{\partial y}$ in (1) obtained by first taking the average of the $E_{x}$-values at $\mathrm{C}$ and $\mathrm{D}$ and at $\mathrm{A}$ and $\mathrm{G}$ resp., and using the resulting values to determine the $y$-derivative in reference point O. Similarly, the r.h.s. of (30) stands for the contribution $\frac{\partial B_{z}}{\partial t}$ in (1). To calculate this time derivative at $t=t_{0}$ in $\mathrm{O}$, first, average $B_{z}$-values in $\mathrm{O}$ are obtained from the values at $\mathrm{E}$ and $\mathrm{B}$ for both $t=t_{0}+\frac{\Delta t}{2}$ and $t=t_{0}-\frac{\Delta t}{2}$. Finally, the second term in the 1.h.s. of (30) is the contribution $\frac{\partial E_{y}}{\partial x}$ in (1) and needs a more careful examination. The approximation for this derivative in $\mathrm{O}$ is obtained using the $E_{y}$-values at $\mathrm{B}, \mathrm{O}$ and $\mathrm{F}$, with weights $-1,0.5$ and 0.5 resp. However, note from Table I that $e_{y}$ at B is discretized at half-integer time instants. As we need the $x$-derivative at an integer time instant, averaging two half-integer time instants intervenes. A similar procedure is adopted for the left interface $\left(x=x_{1}\right)$.

The upper and lower interface conditions are readily retrieved by testing Ampère's law (2) with the HIE testing function (21). Fig. 4 depicts the situation at the upper side $\left(y=y_{2}\right)$ for an arbitrary grid point $\left(x, y_{2}\right)$. To apply (2), we need the value of $B_{z}$ at $\mathrm{O}$. This value is obtained by

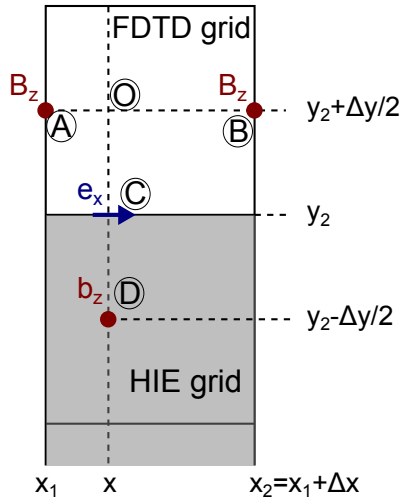

Fig. 4: Scheme relevant to the upper interface condition at $y=y_{2}$ in Fig. 1.

interpolation using the values in $\mathrm{A}$ and $\mathrm{B}$. With this value of $B_{z}$ in $\mathrm{O}$, the value of $b_{z}$ in $\mathrm{D}$ and the value of $e_{x}$ in $\mathrm{C}$, we now apply (22). As this interface will typically be a material boundary as well, the material parameters $\mu, \varepsilon$ and $\sigma$ in (22) need to be averaged over the HIE and FDTD cell. A similar procedure is adopted for the bottom interface $\left(y=y_{1}\right)$.

\section{Implementation}

The HIE-subgridding technique is easily added to existing FDTD codes. In case of a vertically oriented slab (see Fig. 1), the final subgridding algorithm obeys following leapfrog time iteration scheme. First, $B_{z}$ is updated in the FDTD region. The old $B_{z}$ values left and right of the HIE region have to be stored because they are needed for the interface conditions. Next, $e_{y}$ and $b_{z}$ are simultaneously updated in the HIE region using (26). Then, $e_{x}$ is updated in the HIE region using (22). Finally, $E_{x}$ and $E_{y}$ are updated in the FDTD region.

\section{DISPERSION AND STABILITY}

\section{A. Numerical dispersion relation}

As in [26, §4.3], the numerical dispersion of the HIE method is assessed by substituting a discrete plane wave solution with numerical wave vector $\tilde{\boldsymbol{k}}=\tilde{k}_{x} \mathbf{u}_{x}+\tilde{k}_{y} \mathbf{u}_{y}$, but physical frequency $\omega$, into the update equations for vacuum, i.e. (22),

$$
\begin{aligned}
\frac{1}{\Delta y}\left[\frac{1}{2}\right. & \left.\left(e_{x, x_{2}, y_{0}+\Delta y}^{t_{0}}+E_{x, x_{2}+\Delta x, y_{0}+\Delta y}^{t_{0}}\right)-\frac{1}{2}\left(e_{x, x_{2}, y_{0}}^{t_{0}}+E_{x, x_{2}+\Delta x, y_{0}}^{t_{0}}\right)\right] \\
& -\frac{1}{\Delta x}\left[\frac{1}{2}\left(E_{y, x_{2}+\frac{\Delta x}{2}, y_{0}+\frac{\Delta y}{2}}^{t_{0}}+E_{y, x_{2}+\frac{3 \Delta x}{2}, y_{0}+\frac{\Delta y}{2}}^{t_{0}}\right)-\frac{1}{2}\left(e_{y, x_{2}, y_{0}+\frac{\Delta y}{2}}^{t_{0}+\frac{\Delta t}{2}}+e_{y, x_{2}, y_{0}+\frac{\Delta y}{2}}^{t_{0}-\frac{\Delta t}{2}}\right)\right] \\
& =\frac{1}{\Delta t}\left[\frac{1}{2}\left(b_{z, x_{2}, y_{0}+\frac{\Delta y}{2}}^{t_{0}+\frac{\Delta t}{2}}+B_{z, x_{2}+\Delta x, y_{0}+\frac{\Delta y}{2}}^{t_{0}+\frac{\Delta t}{2}}\right)-\frac{1}{2}\left(b_{z, x_{2}, y_{0}+\frac{\Delta y}{2}}^{t_{0}-\frac{\Delta t}{2}}+B_{z, x_{2}+\Delta x, y_{0}+\frac{\Delta y}{2}}^{t_{0}-\frac{\Delta t}{2}}\right)\right]
\end{aligned}
$$

TABLE I: Discretization in time and space in both grids.

\begin{tabular}{c|ccc|cc}
\hline & $\mathbf{3}$ & FDTD & $\mathbf{y}$ & $\mathbf{y}$ & $\mathbf{t}$ \\
\hline $\boldsymbol{E}_{\boldsymbol{x}}$ & $i \Delta x$ & $j \Delta y$ & $n \Delta t$ & $j \Delta y$ & $n \Delta t$ \\
$\boldsymbol{E}_{\boldsymbol{y}}$ & $\left(i+\frac{1}{2}\right) \Delta x$ & $\left(j+\frac{1}{2}\right) \Delta y$ & $n \Delta t$ & $\left(j+\frac{1}{2}\right) \Delta y$ & $\left(n+\frac{1}{2}\right) \Delta t$ \\
$\boldsymbol{B}_{\boldsymbol{z}}$ & $i \Delta x$ & $\left(j+\frac{1}{2}\right) \Delta y$ & $\left(n+\frac{1}{2}\right) \Delta t$ & $\left(j+\frac{1}{2}\right) \Delta y$ & $\left(n+\frac{1}{2}\right) \Delta t$ \\
\hline
\end{tabular}


(24) and (25) with $\varepsilon=\varepsilon_{0}, \mu=\mu_{0}$ and $\sigma=0$. This results in the dispersion relation:

$$
\left(\frac{c \Delta t}{\Delta x} \frac{\tan \left(\frac{\tilde{k}_{x} \Delta x}{2}\right)}{\tan \left(\frac{\omega \Delta t}{2}\right)}\right)^{2}+\left(\frac{c \Delta t}{\Delta y} \frac{\sin \left(\frac{\tilde{k}_{y} \Delta y}{2}\right)}{\sin \left(\frac{\omega \Delta t}{2}\right)}\right)^{2}=1 .
$$

This dispersion relation is not surprising. As was the case for the fully implicit method in [25], the extra interpolations in space and time convert the sine functions of the classical FDTD dispersion relation into tangent functions. Since we apply the implicit technique in the $x$-dimension, only the term corresponding to this dimension features tangent functions. Furthermore, the appearance of solely sine and tangent functions in the dispersion relation is very reassuring: for wellresolved wavelengths, i.e. $\tilde{k_{x}} \Delta x \ll 1$ and $\tilde{k_{y}} \Delta y \ll 1$, and small time step, the numerical solution converts to the physical one, i.e. $k=\omega / c$.

\section{B. Stability}

Since the HIE method consists of both implicit and explicit calculations, the question arises whether the explicit update is bounded by a minimum time step similar to the Courant limit for classical FDTD. In what follows, first, complex-frequency analysis [26, §4.7] is used to see if such limitations exist for the HIE method. In a second step, the matrix stability of the total subgridding technique is investigated.

1) Complex-frequency analysis: Using trigonometric identities, the numerical dispersion relation (34), this time with numerical instead of physical angular frequency, can be transformed into

$$
\sin ^{2}\left(\frac{\tilde{\omega} \Delta t}{2}\right)=\frac{\left(\frac{c \Delta t}{\Delta x} \tan \left(\frac{\tilde{k}_{x} \Delta x}{2}\right)\right)^{2}+\left(\frac{c \Delta t}{\Delta y} \sin \left(\frac{\tilde{k}_{y} \Delta y}{2}\right)\right)^{2}}{\left(\frac{c \Delta t}{\Delta x} \tan \left(\frac{\tilde{k}_{x} \Delta x}{2}\right)\right)^{2}+1} .
$$

If $\xi^{2}$ is defined to be the r.h.s. of (35), one can calculate the numerical frequency as

$$
\tilde{\omega}=\frac{2}{\Delta t} \sin ^{-1}(\xi) .
$$

Note first of all that $\xi$ is a real number. It is observed that $\tilde{\omega}$ can become complex for $|\xi|>1$. In [26, §4.7], it is shown that complex numerical frequencies lead to instabilities. Therefore, numerical stability can only be guaranteed provided that $\xi_{\max } \leq 1$. To determine $\xi_{\max }$, the sine in the r.h.s. of (35) is set equal to one and the tangent terms are replaced by a newly defined variable $\tau$ which can take any nonnegative real value. Further, the 1D Courant number is defined as

$$
S_{y}=\frac{c \Delta t}{\Delta y} .
$$

Now, the aim is to find the value of $\tau$ for which the function

$$
\xi(\tau)=\sqrt{\frac{\tau+S_{y}^{2}}{\tau+1}}, \tau \in \mathbb{R}_{0}^{+}
$$

reaches its maximum. However, taking the derivative and setting it equal to zero gives no solution for $\tau$. In fact, $\xi(\tau)$ is monotonically increasing for $S_{y}<1$, constant for $S_{y}=1$ and monotonically decreasing for $S_{y}>1$. Hence,

$$
\xi_{\max }= \begin{cases}\xi(+\infty)=1 & \text { if } 0 \leq S_{y}<1 \\ 1 & \text { if } S_{y}=1 \\ \xi(0)=S_{y} & \text { if } S_{y}>1\end{cases}
$$

From (39), it is concluded that the stability constraint $\xi_{\max } \leq 1$ corresponds to $S_{y} \leq 1$. As could be expected intuitively, the stability of the HIE method is determined by the 1D Courant limit in the $y$-direction, i.e. the direction in which the explicit update occurs. If $\Delta t$ satisfies the 2D Courant limit, it automatically satisfies the 1D Courant limit as well, so no extra limitations apply to the new subgridding technique.

The stability condition $S_{y} \leq 1$, also has been verified computationally by exciting a HIE grid delimited with perfect electrically conducting (PEC) boundaries for different values of $\Delta t$.

2) Computational analysis of the matrix stability: The complex-frequency analysis is not a sufficient proof for the stability of the HIE-subgridding technique. The classical FDTD method in the coarse grid and the HIE method in the fine grid are both stable if the time step obeys the 2D Courant limit, but yet, nothing is said about the effect of the interfacing between both grids.

As is the case for all time-domain methods, it is possible to express the HIE-subgridding method in terms of one single time-stepping operator. This is a matrix $M$, also called the amplification matrix, that operates on a column vector $V^{n}$ which contains all unknowns at a specific time $t=n \Delta t$, or better said, after a specific number of iterations (since not all unknowns are discretized at the same point in time). The "future" quantities can then be found calculating

$$
V^{n+1}=M V^{n}+U^{n},
$$

where $U^{n}$ is a column vector representing the sources. This describes a linear system, which is known to be BIBO (Bounded-Input Bounded-Output) stable if all eigenvalues of $M$ lie on or inside the unit circle of the complex plane. Eigenvalues inside the unit circle are physically translated to losses, for example due to the presence of a lossy material such as a conductor. Eigenvalues with a small phase angle correspond to temporally well-resolved phenomena, i.e. with many $\Delta t$ per period.

The matrix $M$ can be extracted from a simulation as follows:

1) Define the vector $V^{n}$ containing all unknowns.

2) Assign the unit vector with only the $i^{t h}$ element different from zero to $V^{n}$.

3) Do the necessary calculations to advance one time step.

4) The resulting vector $V^{n+1}$ is the $i^{t h}$ column of $M$.

5) Iterate over all $i$.

Fig. $5 \mathrm{~b}$ shows plots of the eigenvalues $\lambda$ for the following test case (see Fig. 5a). The overall FDTD grid measures 7 by 8 cells with $\Delta x=0.30 \mathrm{~m}$ and $\Delta y=0.25 \mathrm{~m}$. This grid is terminated by PEC and PMC (perfect magnetically conducting) boundaries. The HIE grid covers 4 cells in the 


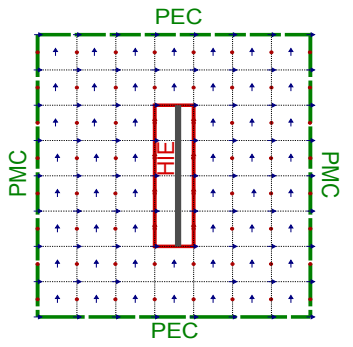

(a)

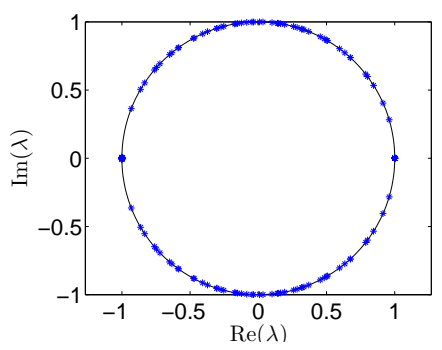

(c)

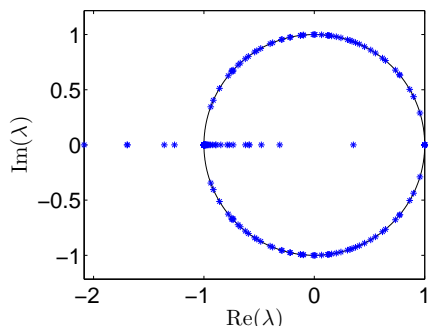

(e)

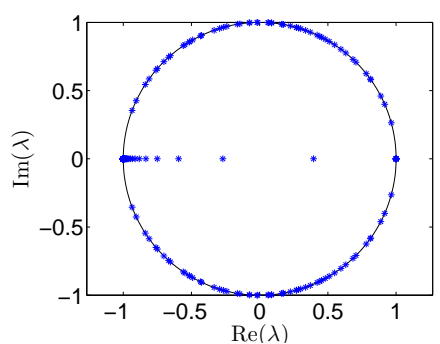

(b)

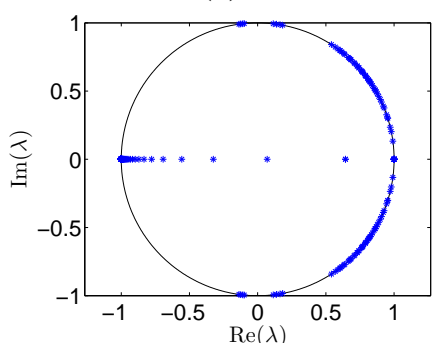

(d)

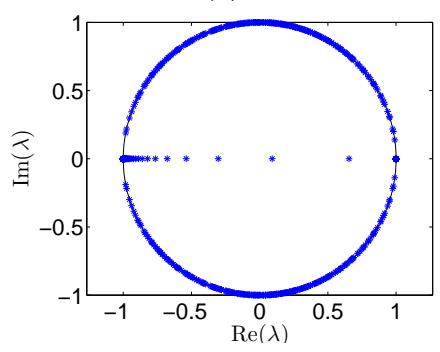

(f)
Fig. 5: Eigenvalues $\lambda$ of the time-stepping operator $M$ for the configuration of (a) with (b) $S_{x y}=1$; (c) $S_{x y}=1$ and vacuum everywhere; (d) $S_{x y}=0.5$; (e) $S_{x y}=1.1$; (f) denser discretization.

$y$-direction and is positioned centrally in the overall grid. In turn, a thin copper plate $\left(\varepsilon_{r}=1, \sigma=5.8 \cdot 10^{7} \mathrm{~S} / \mathrm{m}\right)$ of thickness $10 \mu \mathrm{m}$ is itself positioned slightly towards the right of the center of the HIE grid (at a fraction 0.6 of the HIE region's total width). The discretization step in the $x$-direction for this plate is chosen to be $0.2 \mu \mathrm{m}$ ( 50 divisions over the plate thickness). It should be emphasized that the aspect ratio of the refinement in the $x$-direction exceeds $10^{6}$ ! For this example, the dimension of the matrix $M$ in (40) is $859 \times 859$. The $2 \mathrm{D}$ Courant number

$$
S_{x y}=c \Delta t \sqrt{\frac{1}{\Delta x^{2}}+\frac{1}{\Delta y^{2}}}
$$

is chosen to be 1 . For the other plots, the following modifications were made: $\sigma=0$ (vacuum) in Fig. $5 \mathrm{c}$, a smaller Courant number $\left(S_{x y}=0.5\right)$ in Fig. $5 \mathrm{~d}$ and a larger one $\left(S_{x y}=1.1\right)$ in Fig. 5e. For the vacuum simulation, all eigenvalues are on the unit circle. For a smaller Courant number, i.e. for smaller $\Delta t$, the eigenvalues shift towards lower phase angles, whereas for a Courant number larger than one the simulation becomes unstable, since some eigenvalues are outside the unit circle.

Finally, Fig. 5f presents results similar to Fig. 5b, but for 15 by 16 cells in the overall FDTD grid and the HIE grid covering 8 cells in the $y$-direction, while keeping the overall simulation space unchanged (i.e. the sampling is now denser).

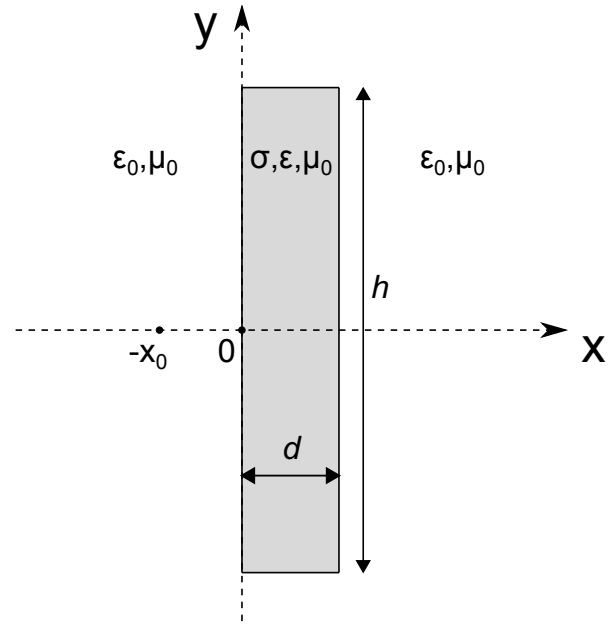

Fig. 6: Set-up for the determination of the transmission through a thin lossy layer.

The thickness of the plate also remains unchanged but is now discretized with a $0.05 \mu \mathrm{m}$ increment (200 divisions over the plate thickness, $M$ has dimension $5801 \times 5801)$. This drastic increase in grid sampling density does not affect the stability.

\section{NUMERICAL EXAMPLES}

\section{A. Transmission of a magnetic line source field through a thin conductive layer}

Consider the geometry of Fig. 6. A conducting slab with thickness $d$ and height $h$ resides in free space. A magnetic line source $\delta\left(x+x_{0}, y\right) g(t) \mathbf{u}_{z}$ illuminates the slab in the near field. To validate our new HIE-subgridding method, simulation results are compared with the analytical results obtained by extending the slab to infinity, i.e. $h \rightarrow \infty$. This analytical solution is derived using a well-known frequencydomain approach [27] for layered media by introducing a spatial Fourier transform of Maxwell's equations along the $y$-coordinate. This yields the magnetic field at the back of the slab $(x=d)$ :

$$
H_{z}^{x=d}(\omega, y)=\frac{1}{2 \pi} \int_{-\infty}^{+\infty} H_{z}^{x=d}\left(\omega, k_{y}\right) e^{j k_{y} y} \mathrm{~d} k_{y}
$$

with

$$
H_{z}^{x=d}\left(\omega, k_{y}\right)=-\frac{2 Z_{1} g(\omega) e^{-j \Gamma_{0} x_{0}}}{\left(Z_{0}+Z_{1}\right)^{2} e^{j \Gamma_{1} d}-\left(Z_{0}-Z_{1}\right)^{2} e^{-j \Gamma_{1} d}}
$$

and $g(\omega)$ the time-domain Fourier transform of $g(t)$. Furthermore, the impedances $Z_{0}$ and $Z_{1}$ are

$$
Z_{0}=\frac{\Gamma_{0}}{\omega \varepsilon_{0}} \quad Z_{1}=\frac{\Gamma_{1}}{\omega \varepsilon_{0} \varepsilon_{r, e f f}},
$$

while the wave numbers $\Gamma_{0}$ and $\Gamma_{1}$ are given by

$$
\Gamma_{0}=\left(k_{0}^{2}-k_{y}^{2}\right)^{\frac{1}{2}} \quad \Gamma_{1}=\left(k_{0}^{2} \varepsilon_{r, e f f}-k_{y}^{2}\right)^{\frac{1}{2}} .
$$

They depend on the vacuum wave number $k_{0}$ and the effective relative permittivity of the conductive slab $\varepsilon_{r, e f f}=\varepsilon_{r}-j \sigma / \omega \varepsilon_{0}$. Both wave numbers must have a phase 


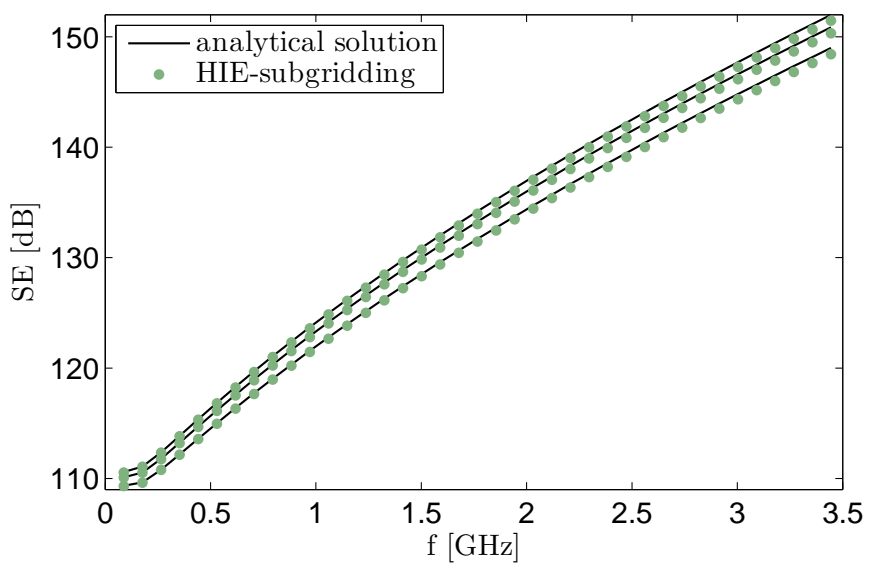

Fig. 7: Shielding effectiveness of a copper slab $(d=10 \mu \mathrm{m}$, $\varepsilon_{r}=1, \sigma=5.8 \cdot 10^{7} \mathrm{~S} / \mathrm{m}$ ) for a near-field H-dipole at $x_{0}=10$ $\mathrm{mm}$. Observation points are placed at $y=0 \mathrm{~mm}$ (highest curve), $y=0.8 \mathrm{~mm}$ and $y=1.6 \mathrm{~mm}$ (lowest curve).

angle in the fourth quadrant in order to ensure the exponentials in (43) to be damped. As a consequence, the integrand of (42) is discontinuous at $k_{y}$-values for which $\Gamma_{0}$ and/or $\Gamma_{1}$ become zero. In order to resolve this behaviour adequately, we use a tanh-sinh quadrature rule [28]. In the absence of the slab, the magnetic field of the line source at $x=d$ is a simple Hankel function

$$
H_{z, \text { vac }}^{x=d}(\omega, y)=-\frac{k_{0}^{2} g(\omega)}{4 \omega \mu_{0}} H_{0}^{(2)}\left(k_{0} \sqrt{\left(x_{0}+d\right)^{2}+y^{2}}\right) .
$$

From the above, we derive the dimensionless shielding effectiveness:

$$
\mathrm{SE}_{\mathrm{dB}}(\omega, y)=20 \log _{10}\left|\frac{H_{z, \text { vac }}^{x=d}(\omega, y)}{H_{z}^{x=d}(\omega, y)}\right| .
$$

For the numerical results discussed below, $\operatorname{SE}(\omega, y)$ is first calculated using the new HIE-subgridding technique. This requires two different runs: one with and one without conductive slab. At every time step, the value of $H_{z}$ is recorded in different observation points along the back of the slab $(x=d)$. The data of both runs are Fourier transformed with respect to $t$ and then divided by each other. The length $h$ is chosen large enough (more specifically, $h>2 c N_{t} \Delta t$, with $N_{t}$ the total number of time iterations) as to ensure that the recorded fields are not yet influenced by diffraction at the top and bottom of the slab. This implies that the numerically obtained $\operatorname{SE}(\omega, y)$ values can be compared to the corresponding analytical values for $h \rightarrow \infty$ obtained by calculating (42).

Fig. 7 shows that the shielding effectiveness of a thin copper slab is accurately computed for all frequencies that are adequately covered by the source. Fig. 8 shows the shielding effectiveness for exactly the same configuration, except for the fact that the slab is made of highly doped silicon.

The simulation details for the copper layer are the following. The time dependence of the line source is

$$
g(t)=\sin \left(2 \pi f_{c} t\right) e^{\frac{-\left(t-t_{d}\right)^{2}}{2 t_{w}^{2}}}
$$

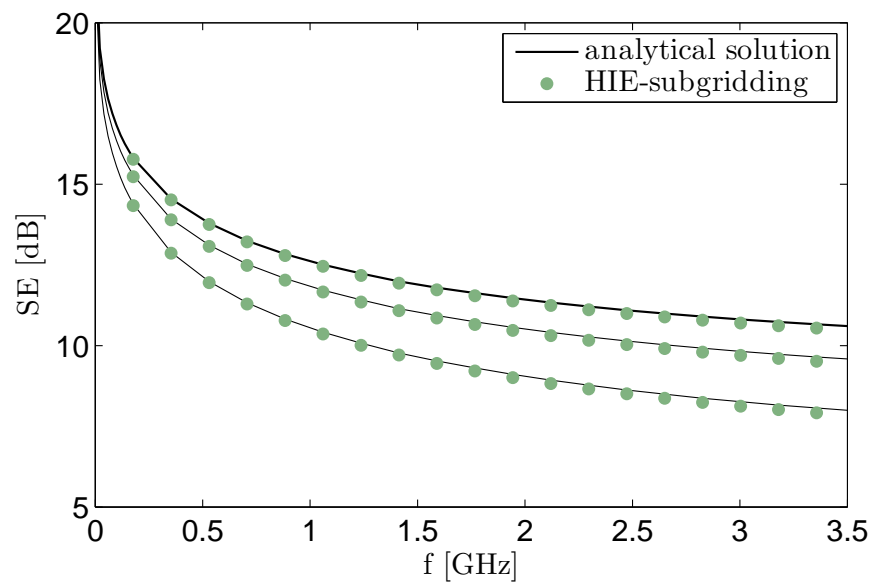

Fig. 8: Shielding effectiveness of a highly doped silicon slab $(d=$ $10 \mu \mathrm{m}, \varepsilon_{r}=11.7, \sigma=10^{3} \mathrm{~S} / \mathrm{m}$ ) for observations on $y=0 \mathrm{~mm}$ (highest curve), $y=0.8 \mathrm{~mm}$ and $y=1.6 \mathrm{~mm}$ (lowest curve).

with $f_{c}=1 \mathrm{GHz}, t_{d}=1 / 4 f_{c}$ and $t_{w}=t_{d} / 2 \sqrt{2 \ln (2)}$. The overall FDTD grid counts 68 by 10202 divisions with $\Delta x=\Delta y=0.8 \mathrm{~mm}$. It is terminated by a 10 -cell thick splitfield perfectly matched layer (PML) [29]. The time step at the Courant limit $\left(S_{x y}=1\right)$ is $\Delta t=1.8869$ ps and the number of time steps is chosen to be $N_{t}=6000$. The HIE subgrid is positioned in the center of the overall FDTD grid. The copper slab inside the HIE grid has width $d=10 \mu \mathrm{m}$ and counts 50 by 10180 cells, which also corresponds to the segmentation of the HIE grid except for the fact that there are two more divisions in the $x$-direction to set up the padding area (see Fig. 1). Hence, the height of the slab is $h=10180 \Delta y=8.144 \mathrm{~m}$ and the refinement ratio in the $x$-direction for the cells inside the slab is $50 \Delta x / d=4000$. The slab's left surface is exactly in the middle of the HIE grid so that the source, which is placed 12 cells to the left of the HIE grid, is $10 \mathrm{~mm}$ removed from the slab, i.e. $x_{0}=10 \mathrm{~mm}$. With these choices, diffraction effects at the upper and lower edges of the copper slab do not influence the presented data.

For the silicon slab, the same parameters are used but now the FDTD grid and the slab have respectively 5112 and 5090 divisions in the $y$-direction, and $N_{t}=3000$.

As remarked by one of the reviewers, in the past, thin conductive sheets have already attracted a lot of attention in the FDTD community. We refer the reader to [30] for a recent contribution and to the list of related papers cited in the references of [30]. In [30], the application of the impedance network boundary conditions (INBC) together with a new approach to treat the so-called H-node shift problem, is shown to lead to a very good prediction of the shielding effectiveness. In the present paper, the thin sheet example only serves as a way to illustrate the accuracy of the proposed method (with a maximum error for the SE of $0.4 \mathrm{~dB}$ in the tail of the source's spectrum). In contrast to the INBC approach, HIE-subgridding is not restricted to good and homogeneous conductors $(\sigma \gg \omega \varepsilon)$, but can handle any type of material. It does not depend on the fact that a 1D thin sheet approximation is introduced (see e.g. the grating example below). 


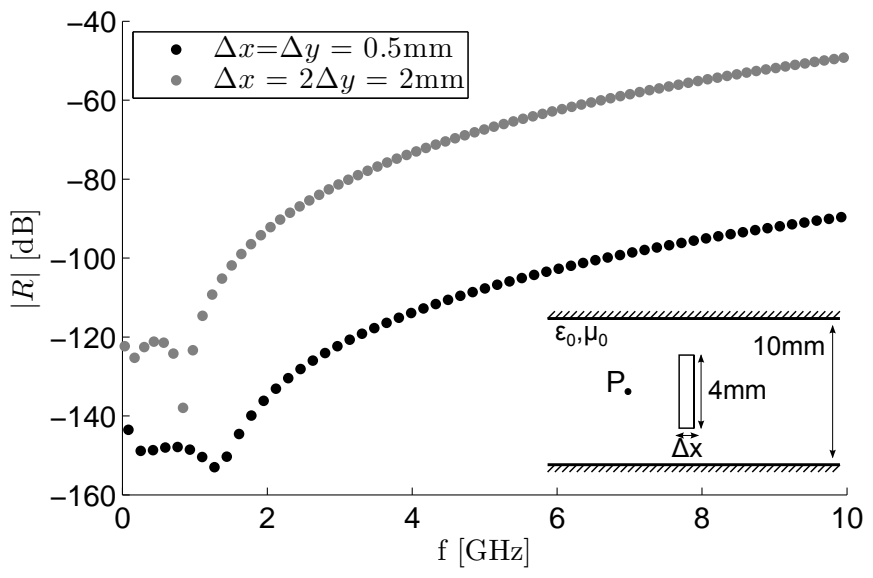

Fig. 9: Quantization of spurious reflections: magnitude of the reflection coefficient of a vacuum HIE-subgrid in a vacuum parallel plate waveguide excited by its TEM mode. The physical configuration is presented as an inset.

\section{B. Vacuum simulation and spurious reflections}

In analogy to $[3,31]$, the spurious reflections originating from the interface between the coarse FDTD grid and the HIE-subgrid are quantified by exciting a vacuum parallel plate waveguide by a TEM pulse which propagates towards a vacuum HIE-subgrid. The configuration is shown in the inset of Fig. 9. The separation between the two plates is $10 \mathrm{~mm}$, the subgrid measures $4 \mathrm{~mm}$ in the $y$-direction and has a thickness $\Delta x$ of one coarse FDTD cell. The HIE-subgrid consists of 50 subdivisions in the $x$-dimension with $\Delta x_{f}=0.2 \mu \mathrm{m}$, completed by two padding areas in order to obtain one $\Delta x$. The magnitude of the reflection coefficient

$$
R(\omega)=\frac{H_{z}^{\text {tot }}(\omega)-H_{z}^{i n c}(\omega)}{H_{z}^{i n c}(\omega)}
$$

is plotted in Fig. 9 for two coarse grid cell sizes: $\Delta x=\Delta y=0.5 \mathrm{~mm}$ and $\Delta x=2 \Delta y=2 \mathrm{~mm}$. The total field $H_{z}^{\text {tot }}(t)=H_{z}^{\text {inc }}(t)+H_{z}^{\text {refl }}(t)$ is recorded in an observation point $\mathrm{P}$ placed five cells in front of the subgrid. An auxiliary simulation without subgrid provides the incident field values $H_{z}^{i n c}(t)$. Fig. 9 confirms that HIE-subgridding can easily compete with other subgridding methods such as the conservative subgridding scheme described in [31]. As the frequency increases, the sampling density of the grid relative to the wavelength drops and the discrepancy between the dispersion relations in both grids augments, which unavoidably results in higher reflections. For the same reason, the coarser discretization of the FDTD region results in a higher reflection coefficient.

\section{Skin effect}

One of the purposes of the new subgridding method is to accurately model thin good conductor effects. We again turn to the set-up of Fig. 6, but now with a finite slab having $h=10 \mathrm{~mm}$. The slab is made of copper and has thickness $d=10 \mu \mathrm{m}$. It is discretized with a $0.2 \mu \mathrm{m}$ increment in the $x$-direction. The FDTD cell dimensions are $\Delta x=0.253 \mathrm{~mm}$ and $\Delta y=0.250 \mathrm{~mm}$. The source is again a

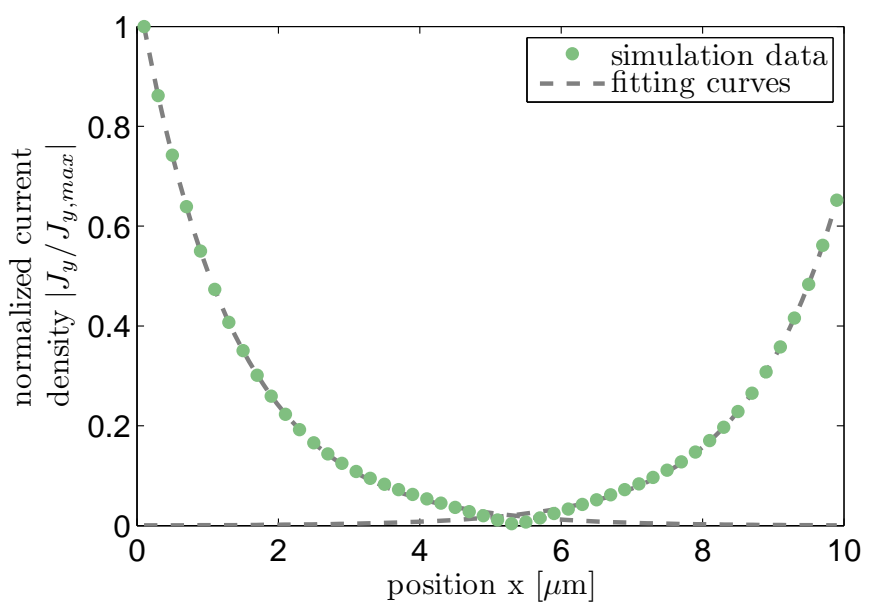

Fig. 10: Current density in the cross section of a thin copper slab $\left(d=10 \mu \mathrm{m}, h=10 \mathrm{~mm}, \sigma=5.8 \cdot 10^{7} \mathrm{~S} / \mathrm{m}\right)$ at $f=2.45 \mathrm{GHz}$.

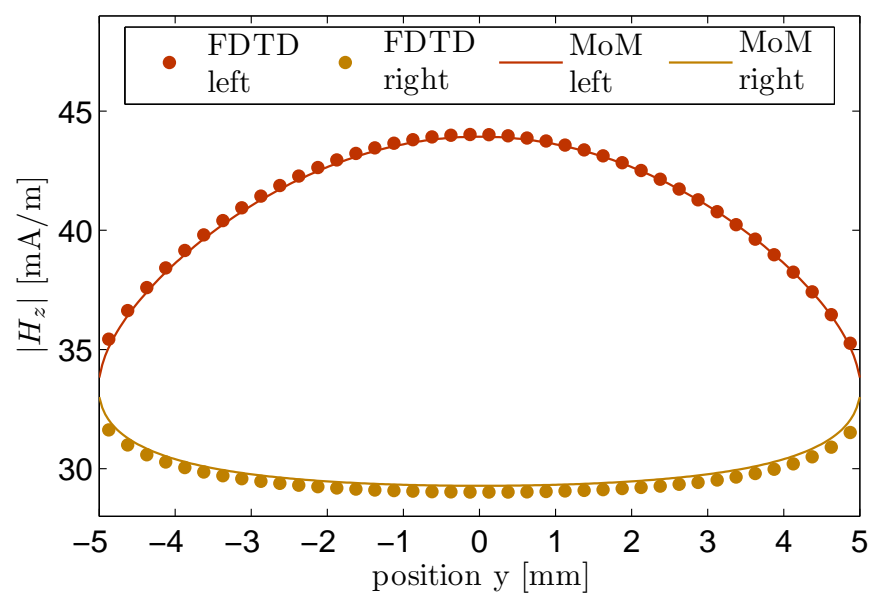

Fig. 11: Field solutions on the slab surface compared to MoM.

Gaussian-modulated sinusoidal pulse (48) placed at a distance $x_{0}=10 \mathrm{~mm}$ from the front of the copper slab. Fig. 10 displays the normalized magnitude of the current density in the $y=0$-cross-section at $2.45 \mathrm{GHz}$ (green dots). At this frequency, the skin depth $\delta$ is $1.3351 \mu \mathrm{m}$. Two exponentials are fitted (in the least-squares sense) to the data points that lie within one skin depth distance from the left and right conductor surface, resulting in the dashed lines in Fig. 10. The fitting leads to the following numerical data for the skin depth: $\delta_{\text {left }}=1.3353 \mu \mathrm{m}$ and $\delta_{\text {right }}=1.3342 \mu \mathrm{m}$, i.e. a relative error of less than one per thousand.

\section{Field solutions on the material surface compared to MoM}

For exactly the same set-up as in Section IV-C, the magnetic fields along the left and right surface of the copper slab are compared to the output of Nero2d [32], a noncommercial MoM-solver. Fig. 11 again confirms the accuracy of the HIEsubgridding technique.

\section{E. Radiation pattern of a metal grating structure}

As a last example, the far-field radiation pattern of a metal grating is studied. The set-up is shown in Fig. 12: an incident 


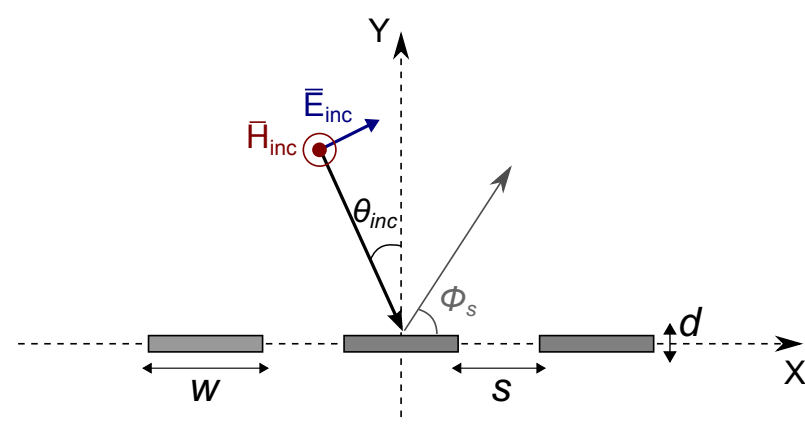

Fig. 12: A plane TE-wave incident on a 3-strip metal grating.

plane TE-wave impinges upon a number of metal strips under an angle $\theta_{\text {inc }}$. The incident-field array (IFA) excitation is applied in combination with the total-field/scattered-field formalism [26, §5.7]. Fourth-order interpolation as well as high grid sampling densities (at least 30 cells per wavelength) are used to enhance the accuracy of the plane wave excitation. The scattered-field region is enveloped by classical split-field PMLs. The metal grating structure behaves like a phased antenna array. Its far-field radiation pattern can be determined by applying a near-to-far-field (NTFF) transformation. Thereto, we adopt the frequency-domain approach described in [33, §8.3.2]. Fig. 13 shows the scattering width, i.e.

$$
\sigma_{2 D}=\lim _{\rho \rightarrow \infty} 2 \pi \rho\left|\frac{E_{\phi}(\rho)}{E_{\text {inc }}}\right|^{2},
$$

for a 3-strip metal grating, illuminated by a $2.45 \mathrm{GHz}$ plane wave incident under an angle $\theta_{i n c}=30^{\circ}$, for various values of the electrical conductivity. The scattering is strongest in the specular direction $\phi_{s}=60^{\circ}$. Furthermore, according to the antenna array factor, one expects to see a grating lobe at $\phi_{s}=\arccos \left(\sin \left(\theta_{i n c}\right)-\frac{\lambda}{w+s}\right)$, which is for $\theta_{i n c}=30^{\circ}$, $w=80 \mathrm{~mm}$ and $s=10 \mathrm{~mm}$ equal to $149.27^{\circ}$ (with $\lambda$ the wavelength of the incident wave). This additional grating lobe becomes more clear for a higher number of strips, as can be seen in Fig. 14. For a $2.45 \mathrm{GHz}$ source, the skin depth equals the thickness of the strips, i.e. $\delta=d=0.5 \mathrm{~mm}$, when $\sigma=413.6 \mathrm{~S} / \mathrm{m}$. In Fig. 13, this is translated to a fast increase in the amount of scattered radiation between an electrical conductivity of 1 and $1000 \mathrm{~S} / \mathrm{m}$. For higher values of $\sigma$, the radiation pattern remains (almost) identical.

\section{CONCLUSiON}

We proposed a new thin-layer subgridding technique for 2D-TE problems based on a hybrid implicit-explicit scheme. This new technique allows very fine sampling in the direction perpendicular to the thin layer, making it, e.g., ideally suited to model the skin effect. The implicit part of the HIE-subgridding method, applied inside the subgrid, leverages a collocated discretization scheme, as such avoiding any ambiguity concerning the thickness of the thin material layers. Furthermore, it permits nonuniform discretizations without jeopardizing the stability. This stability was thoroughly analyzed and the classical 2D Courant limit, imposed by the coarse FDTD grid, is found to be the only restriction. Regarding the implementation, the subgridding technique is easily integrated into existing

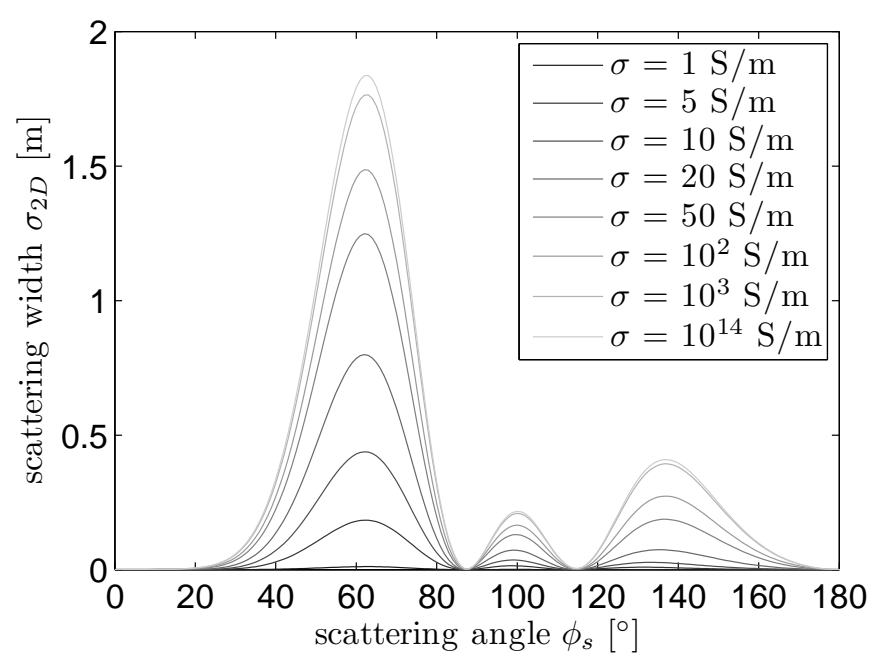

Fig. 13: Radiation pattern of a 3-strip metal grating ( $w=80 \mathrm{~mm}$, $s=10 \mathrm{~mm}, d=0.5 \mathrm{~mm}$ ) for different values of the conductivity $\sigma$ $\left(\varepsilon_{r}=1\right)$.

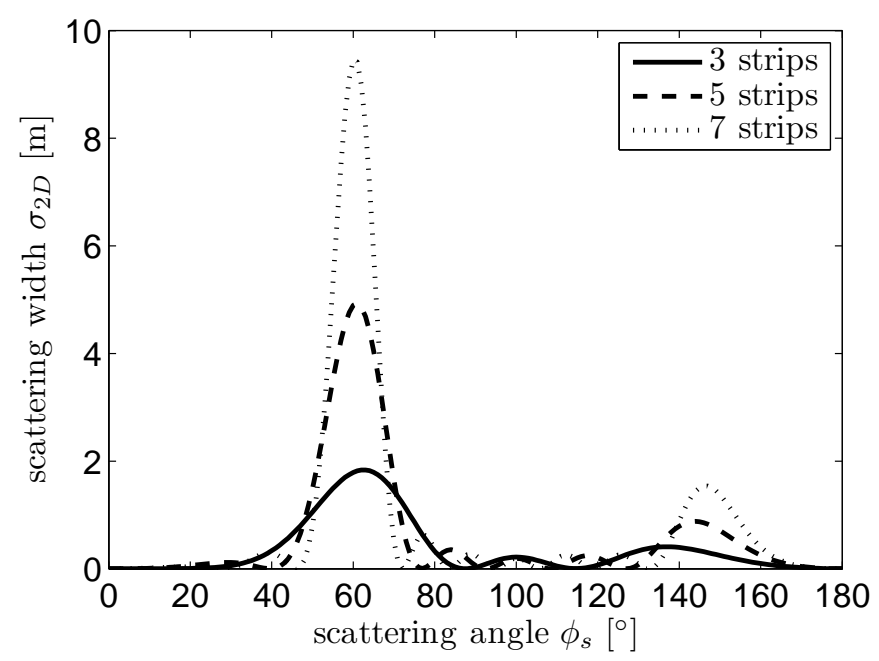

Fig. 14: Radiation pattern of a copper grating structure $(w=80 \mathrm{~mm}$, $s=10 \mathrm{~mm}, d=0.5 \mathrm{~mm}, \varepsilon_{r}=1, \sigma=5.8 \cdot 10^{7} \mathrm{~S} / \mathrm{m}$ ) illuminated by a $2.45 \mathrm{GHz}$ plane wave incident under an angle $\theta=30^{\circ}$ for various numbers of strips.

FDTD codes as it only requires some extra steps in the leapfrog time iteration scheme in conjunction with minor modifications to the FDTD code itself. The novel hybrid scheme's accuracy is validated by means of several numerical examples, showing, e.g., that the substantial effect of finite conductivity on the power scattered by a metal grating can be accurately predicted. Future work will focus on an analogous subgridding scheme for the TM-case and for more general electromagnetic problems in the full three-dimensional space, as well as a PML formulation for the HIE method allowing the efficient simulation of infinitely long thin layers.

\section{APPENDIX}

The goal of this appendix is to give a mathematical derivation of the discrete form of Ampère's law (25) as used by the HIE scheme. Thereto, Ampère's law (3) is expanded in basis functions according to (19)-(20), and then tested by (23). The 
testing step leads to the following set of integrals that have to be calculated. For testing of the 1.h.s. of (3), we need

$$
\begin{aligned}
\int \frac{\partial}{\partial x}\left(\Lambda_{i}(x)\right) \Pi_{k+\frac{1}{2}}(x) \mathrm{d} x & =\delta_{i, k+1}-\delta_{i, k} \\
\int \Pi_{j+\frac{1}{2}}(y) \Pi_{l+\frac{1}{2}}(y) \mathrm{d} y & =\Delta y \delta_{j, l} \\
\int \Pi_{n+\frac{1}{2}}(t) \Lambda_{m}(t) \mathrm{d} t & =\frac{\Delta t}{2}\left(\delta_{n, m}+\delta_{n, m-1}\right)
\end{aligned}
$$

For testing the r.h.s. of (3), we need

$$
\begin{aligned}
\int \Lambda_{i}(x) \Pi_{k+\frac{1}{2}}(x) \mathrm{d} x & =\frac{\Delta x_{f}}{2}\left(\delta_{i, k+1}+\delta_{i, k}\right) \\
\int \Pi_{j+\frac{1}{2}}(y) \Pi_{l+\frac{1}{2}}(y) \mathrm{d} y & =\Delta y \delta_{j, l} \\
\int \frac{\partial}{\partial t}\left(\Pi_{n+\frac{1}{2}}(t)\right) \Lambda_{m}(t) \mathrm{d} t & =\delta_{n, m}-\delta_{n, m-1} \\
\int \Pi_{n+\frac{1}{2}}(t) \Lambda_{m}(t) \mathrm{d} t & =\frac{\Delta t}{2}\left(\delta_{n, m}+\delta_{n, m-1}\right)
\end{aligned}
$$

The integrals evaluate to Kronecker deltas. Inserting these solutions in the expanded form of (3), and subsequently dividing both sides of the resulting discrete equation by $\frac{\Delta x_{f} \Delta y \Delta t}{2}$, eventually gives (25).

\section{REFERENCES}

[1] B. Donderici and F. Teixeira, "Improved FDTD Subgridding Algorithms via Digital Filtering and Domain Overriding,", IEEE Transactions on Antennas and Propagation, vol. 53, no. 9, pp. 2938-2951, September 2005.

[2] F. Collino, T. Fouquet, and P. Joly, "Conservative space-time mesh refinement methods for the FDTD solution of Maxwell's equations," Journal of Computational Physics, vol. 211, no. 1, pp. 9-35, January 2006.

[3] R. Chilton, "H-, P- and T-refinement strategies for the Finite-DifferenceTime-Domain (FDTD) method developed via Finite-Element (FE) principles," Ph.D. dissertation, Ohio State University, 2008.

[4] J.-P. Bérenger, "Extension of the FDTD Huygens Subgridding Algorithm to Two Dimensions," IEEE Transactions on Antennas and Propagation, vol. 57, no. 12, pp. 3860-3867, December 2009.

[5] _ - "The Huygens subgridding for the numerical solution of the Maxwell equations," Journal of Computational Physics, vol. 230, no. 14, pp. 5635-5659, June 2011.

[6] C. Chang and C. Sarris, "A Spatially Filtered Finite-Difference TimeDomain Scheme with Controllable Stability Beyond the CFL Limit: Theory and Applications," IEEE Transactions on Microwave Theory and Techniques, vol. 61, no. 1, pp. 351-359, January 2013.

[7] _ , "An Efficient Implementation of a 3D Spatially-Filtered FDTD Subgridding Scheme," in IEEE Microwave Symposium Digest (MTT), Chicago, July 2012, pp. 1-2.

[8] M. Gaffar and D. Jiao, "An Explicit and Unconditionally Stable FDTD Method for Electromagnetic Analysis," IEEE Transactions on Microwave Theory and Techniques, vol. 62, no. 11, pp. 2538-2550, November 2014.

[9] G. Sun and C. Trueman, "Unconditionally stable Crank-Nicolson scheme for solving two-dimensional Maxwell's equations," Electronic Letters, vol. 39, no. 7, pp. 595-597, April 2003.

[10] F. Zheng, Z. Chen, and J. Zhang, "A Finite-Difference Time-Domain Method Without the Courant Stability Conditions," IEEE Microwave and Guided Wave Letters, vol. 9, no. 11, pp. 441-443, November 1999.

[11] S. J., M. Muraki, J. Yamauchi, and H. Nakano, "Efficient implicit FDTD algorithm based on locally one-dimensional scheme," Electronics Letters, vol. 41, no. 19, September 2005.

[12] T. Hemmi, F. Costen, S. Garcia, R. Himeno, H. Yokota, and M. Mustafa, "Efficient Parallel LOD-FDTD Method for Debye-Dispersive Media," IEEE Transactions on Antennas and Propagation, vol. 62, no. 3, pp. 1330-1338, March 2014.

[13] Y.-S. Chung, T. Sarkar, B. Jung, and M. Salazar-Palma, "An Unconditionally Stable Scheme for the Finite-Difference Time-Domain Method," IEEE Transactions on Microwave Theory and Techniques, vol. 51, no. 3, pp. 697-704, March 2003.
[14] M. Ha, K. Srinivasan, and M. Swaminathan, "Transient Chip-Package Cosimulation of Multiscale Structures Using the Laguerre-FDTD Scheme," IEEE Transactions on Advanced Packaging, vol. 32, no. 4, pp. 816-830, November 2009.

[15] J. Shibayama, M. Muraki, R. Takahashi, J. Yamauchi, and H. Nakano, "Performance Evaluation of Several Implicit FDTD Methods for Optical Waveguide Analyses," Journal of Lightwave Technology, vol. 24, no. 6, pp. 2465-2471, June 2006.

[16] V. Nascimento, K.-Y. Jung, B.-H. Borges, and F. Teixeira, "A Study on Unconditionally Stable FDTD Methods for the Modeling of Metamaterials," Journal of Lightwave Technology, vol. 24, no. 6, pp. 4241-4249, October 2009

[17] A. Grande and J. Pereda, "Accuracy Limitations of the Locally OneDimensional FDTD Technique," IEEE Antennas and Wireless Propagation Letters, vol. 13, pp. 1180-1183, 2014.

[18] B.-Z. Wang, Y. Wang, W. Yu, and R. Mittra, "A Hybrid 2-D ADIFDTD Subgridding Scheme for Modeling On-Chip Interconnects," IEEE Transactions on Advanced Packaging, vol. 24, no. 4, pp. 528-533, November 2001.

[19] I. Ahmed and Z. Chen, "A Hybrid ADI-FDTD Subgridding Scheme for Efficient Electromagnetic Computation," International Journal of Numerical Modeling: Electonic Networks, Devices and Fields, vol. 17, pp. 237-249, 2004.

[20] Z. Huang, G. Pan, and K.-S. Chen, "A Synchronized Multigrid Time Domain Method Via Huygens Subgridding and Implicit Algorithms," IEEE Transactions on Antennas and Propagation, vol. 61, no. 5, pp. 2605-2614, May 2013.

[21] J. Chen and J. Wang, "A 3D Hybrid Implicit-Explicit FDTD Scheme with Weakly conditional stability," Microwave and Optical Technology Letters, vol. 48, no. 11, pp. 2291-2294, November 2006.

[22] Q. Zhang, B. Zhou, and J. Wang, "A Novel Hybrid Implicit-Explicit FDTD Algorithm with More Relaxed Stability Condition," IEEE Antennas and Wireless Propagation Letters, vol. 12, pp. 1372-1375, October 2013.

[23] W. Tierens, "Finite Element and Finite Difference Based Approaches for the Time-Domain Simulation of Plasma-Wave Interactions," Ph.D. dissertation, Ghent University, 2013.

[24] W. Tierens and D. De Zutter, "Implicit Local Refinement for Evanescent Layers Combined with Classical FDTD," IEEE Microwave and Wireless Components Letters, vol. 23, no. 5, pp. 225-227, May 2013.

[25] _ "An unconditionally stable time-domain discretization on cartesian meshes for the simulation of nonuniform magnetized cold plasma," Journal of Computational Physics, vol. 231, pp. 5144-5156, April 2012.

[26] A. Taflove and S. Hagness, Computational Electrodynamics: The FiniteDifference Time-Domain Method (3th edition). Artech House, 2005.

[27] N. Faché and D. De Zutter, "Full-wave analysis of a perfectly conducting wire transmission line in a double-layered conductor-backed medium," IEEE Transactions on Microwave Theory and Techniques, vol. 37, no. 3, pp. 512-518, March 1989.

[28] D. Bailey, J. Borwein, N. Calkin, R. Girgensohn, R. Luke, and V. Moll, Experimental Mathematics In Action. A. K. Peters, 2006.

[29] J.-P. Bérenger, "A Perfectly Matched Layer for the Absorption of Electromagnetic Waves," Journal of Computational Physics, vol. 114, no. 2, pp. 185-200, October 1994.

[30] V. Nayyeri, M. Soleimani, and O. Ramahi, "A method to model thin conductive layers in the finite-difference time-domain method," IEEE Transactions on Electromagnetic Compatibility, vol. 56, no. 2, pp. 385392, April 2014.

[31] R. Chilton and R. Lee, "Conservative and Provably Stable FDTD Subgridding," IEEE Transactions on Antennas and Propagation, vol. 55, no. 9, pp. 2537-2549, September 2007.

[32] D. Dobbelaere, H. Rogier, and D. De Zutter, "Accurate 2.5-D boundary element method for conductive media," Radio Science, vol. 49, no. 6, pp. 389-399, June 2014.

[33] S. Gedney, Introduction to the Finite-Difference Time-Domain (FDTD) Method for Electromagnetics. Morgan \& Claypool, 2011. 


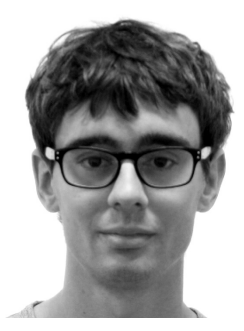

Arne Van Londersele was born in 1991 in Aalst, Belgium. He received the M.Sc. degree in engineering physics from Ghent University, Gent, Belgium, in 2014. Since August 2014, he has been pursuing the Ph.D. degree at the IBCN/Electromagnetics Group, Department of Information Technology, Ghent University/iMinds. His primary research concerns computational electromagnetics and, more specifically, subgridding techniques for the finitedifference time-domain method.

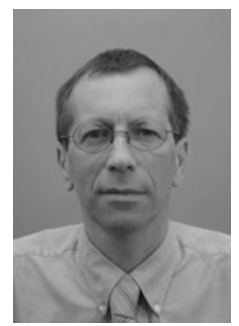

Daniël De Zutter (F'00) was born in 1953. He received his M.Sc. Degree in electrical engineering from Ghent University in 1976. In 1981 he obtained a Ph.D. degree and in 1984 he completed a thesis leading to a degree equivalent to the French Aggrgation or the German Habilitation. He is now a full professor of electromagnetics. His research focusses on all aspects of circuit and electromagnetic modelling of high-speed and highfrequency interconnections and packaging, on Electromagnetic Compatibility (EMC) and numerical solutions of Maxwells equations. As author or co-author he has contributed to more than 200 international journal papers (cited in the Web of Science) and 200 papers in conference proceedings. In 2000 he was elected to the grade of Fellow of the IEEE. He was an Associate Editor for the IEEE Microwave Theory and Techniques Transactions. Between 2004 and 2008 he served as the Dean of the Faculty of Engineering of Ghent University and is now the head of the Department of Information Technology.

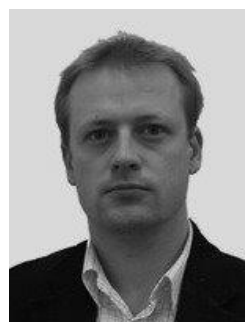

Dries Vande Ginste (SM'12) received the M.S. degree and the Ph.D. degree in electrical engineering from Ghent University, Gent, Belgium, in 2000 and 2005, respectively. He is currently an Associate Professor with the Department of Information Technology, Ghent University and a Guest Professor at iMinds. In June and July 2004, he was a Visiting Scientist at the Department of Electrical and Computer Engineering, University of Illinois at UrbanaChampaign (UIUC), IL, USA. From September to November 2011, he was a Visiting Professor at the EMC Group, Dipartimento di Elettronica, Politecnico di Torino, Italy. $\mathrm{He}$ has authored or co-authored over 100 papers in international journals and in conference proceedings. His research interests include computational electromagnetics, electromagnetic compatibility, signal and power integrity, and antenna design. Dr. Vande Ginste was awarded the International Union of Radio Science (URSI) Young Scientist Award at the 2011 URSI General Assembly and Scientific Symposium, the Best Poster Paper Award at the 2012 IEEE Electrical Design of Advanced Packaging and Systems Symposium (EDAPS), the Best Paper Award at the 2013 IEEE Workshop on Signal and Power Integrity (SPI) and the Best Paper Award at the 2013 IEEE International Conference on Electrical Performance of Electronic Packaging and Systems (EPEPS). He served as the co-chair of the 2014 IEEE Workshop on Signal and Power Integrity. He is a Senior Member of the IEEE. 